\title{
Bringing ACRL Home: The Chapters
}

ACRL chapters (there are twenty-one of them) cover twenty-seven states and one Canadian province. The chapters provide a framework, close to home, for librarians to share ideas, study common problems, and meet for social and professional purposes.

ACRL chapters hold program meetings, sponsor workshops, publish newsletters, undertake studies, and promote legislative programs at the grass-roots level.

So far during 1979, chapters have sponsored program meetings on such topics as: "Economic Reality: Strategies for the Academic Library," "Is Anyone out There Listening? Communications and the Academic Library," and "Interpreting On-Line Reference Services in Libraries, "

The ACRL national organization has begun a new program to encourage chapter activities. Each chapter is now eligible to receive $\$ 1.50$ from ACRL for each national personal member living within the geographic area served by the chapter.

To join an ACRL chapter, find the chapter that serves your area on the list provided. Write the chapter president for information on membership and chapter activities.

To form an ACRL chapter where none exists or to affiliate an existing organization of college and research librarians with $\mathrm{ACRL}$, you may petition the ACRL board for chapter status. For more information on establishing a chapter, see "Guidelines for ACRL Chapters," in the November 1978 issue of CLRL News, or contact the executive secretary, ACRL.

\section{ACRL Chapters}

\section{Delaware Valley Chapter}

President: Mary Nehlig, Francis Harvey Green Library, West Chester State College, High St. \& Rosedale Ave., West Chester, PA 19380

\section{Eastern New York Chapter}

President: Catherine Chmura, Siena College Library, Loudonville, NY 12211

\section{Florida Chapter}

Chair: Dr. John Goudeau, Professor, School of Library Science, Florida State University, Tallahassee, FL 32306

\section{Illinois Chapter}

President: Frances M. Pollard, Chair, Department of Library Science, Eastern Illinois University, Charleston, IL 61920

\section{Iowa Chapter}

Chair: Charles Le Master, Morningside College, Sioux City, IA 51106

\section{Kansas Chapter}

Chair: Virginia Quiring, Assistant Director,
Farrell Library, Kansas State University, Manhattan, KS 60506

\section{Maryland Chapter}

Chair: Mary Eidleman, Coordinator of Library Services, Dundalk Community College, 7200 Sollers Point Rd., Baltimore, MD 21222

\section{Michigan Chapter}

President: Mary George, Bibliographic Instructor and Reference Librarian, Graduate Library, University of Michigan, Ann Arbor, MI 48103

\section{Minnesota Chapter}

Chair: Forrest Brown, Rolvaag Memorial Library, St. Olaf College, Northfield, MN 55057

\section{Missouri Chapter}

President: Joanna Todd, Stephens College Library, Columbia, MO 65201

\section{New England Chapter}

President: Patricia G. Oyler, Assistant Professor, Simmons College, School of Library Science, 300 The Fenway, Boston, MA 02115

\section{North Carolina Chapter}

Chair: Elvin E. Strowd, William R. Perkins Library, Duke University, Durham, NC 27706

Northern California Chapter

Acting Chair: George M. Bailey, The Honnold Library, Claremont, CA 91711

\section{Oregon Chapter}

Chair: Jenko Lukac, The Library, Pacific University, Forest Grove, OR 97116

\section{Southern California Chapter}

Chair: Judith M. Corin, University of California, Los Angeles, Library, 405 Hilgard Ave., Los Angeles, CA 90024

\section{Tennessee Chapter}

Chair: Joe Rader, Undergraduate Library, Reserve, University of Tennessee, Knoxville, TN 37916

\section{Texas Chapter}

Chair: Imogene Gibson, Librarian, Austin College, Hopkins Library, Sherman, TX 75090

\section{Tri-State Chapter}

President: Mary Alice Hupp, Fairmont State College Library, Locust Ave., Fairmont, West Virginia 26554

\section{Virginia Chapter}

Chair: Dennis E. Robison, Chairman, University of Richmond Library, Richmond, VA 23173

\section{Wisconsin Chapter}

Chair: John J. Jax, Pierce Library, University of Wisconsin-Stout, Menomonie, WI 54751

\section{Western New York/Ontario Chapter}

President: Sheila Laidlaw, Director, Sigmund Samuel Library, University of Toronto, Toronto, ON M5S 1A5, Canada 


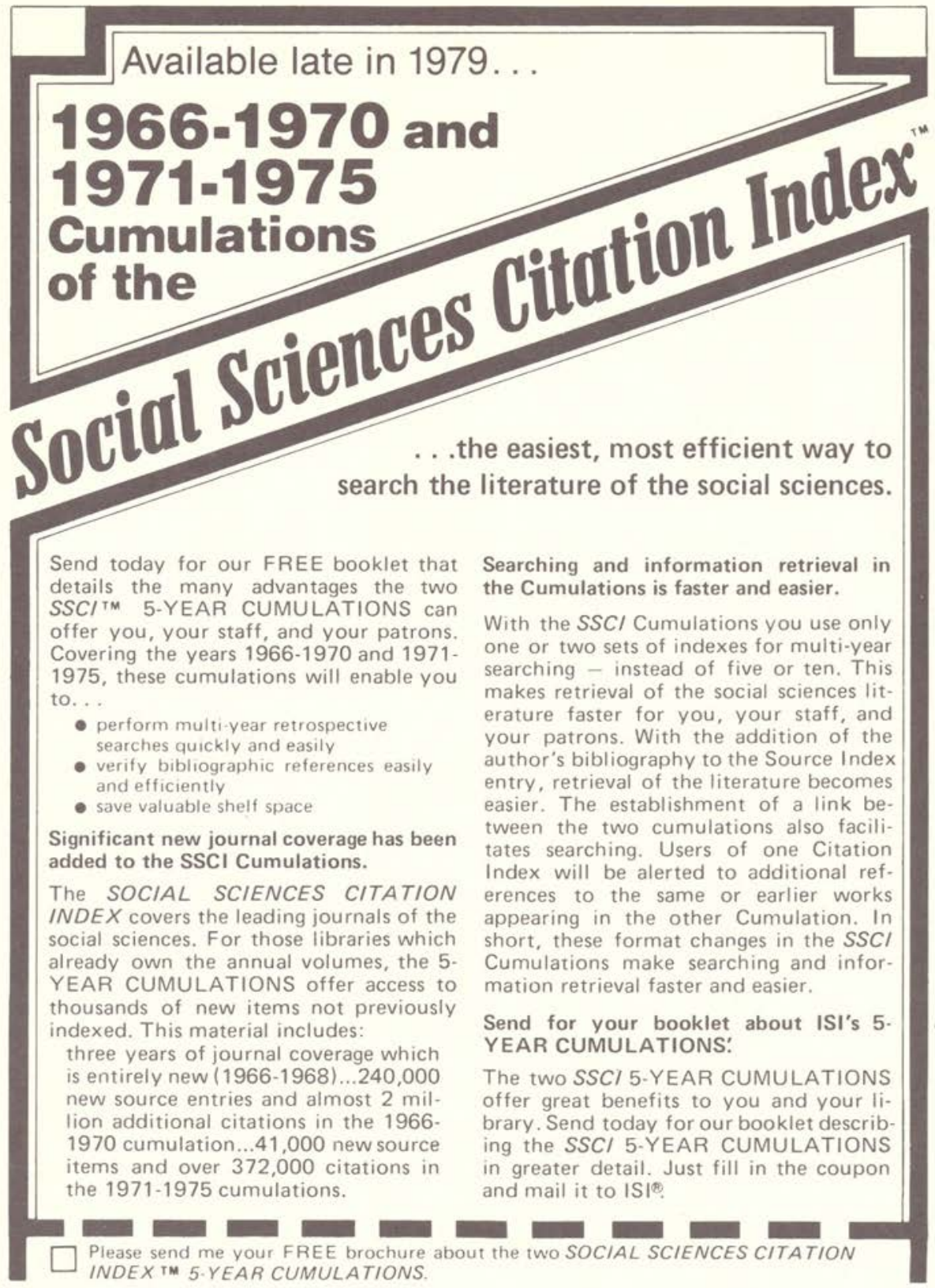

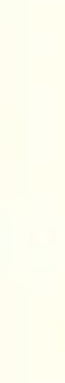
INDEX TM 5.YEAR CUMULATIONS.

Name Title

Institution Dept.

Address

City State/Province ZIP 只 $20^{8}$ Institute for Country 325 Chestnut Street, Philadelphia, Pa. 19106, U.S A Tel: (215) 923-3300, Cable: SCINFO. Telex: 84-5305 Phone 